\title{
Abstracts From the Third Annual Medicine \& Science in Ultra- Endurance Sports Conference, August 2016, Chamonix, France
}

\section{August 21, 2016}

\section{4:00-14:10}

Right Ventricle Adaptation After an Endurance Race

Maria Sanz De La Garza, MD¹; Daniel Brotons Cuixart, MD, $\mathrm{PhD}^{2}$; Gonzalo Gazioli, $\mathrm{MD}^{1}$; Emma Roca, $\mathrm{MD}^{3}$; Marta Sitges, $\mathrm{MD}, \mathrm{PhD}^{1}$

${ }^{1}$ Thorax Institute. Hospital Clinic, IDIBAPS, University of Barcelona, Spain, ${ }^{2}$ Catalan Sports Council, Barcelona, Spain, ${ }^{3}$ University of Girona, Spain

Objective.-Right ventricle (RV) dysfunction and changes in the pulmonary pressure has been described in athletes after endurance races. We aimed to understand the changes in the right heart response to endurance exercise, and the effects of the amount of exercise. Methods.-Echocardiography was performed in 55 healthy adults at baseline and after a three-stage trail race: short (14 km; $\mathrm{n}=17)$; medium (35 km; $\mathrm{n}=21)$; and long (56 km; $\mathrm{n}$ =17). Echocardiographic assessment of the RV was performed with global and separate analysis of the RV basal and apical regions. Results.-No changes were observed in short-distance runners, RV systolic deformation decreased significantly $(\mathrm{p}<0.05)$ after both the medium-length and long races ( $\Delta \%$ RV global strain: $-7.6 \pm 20.1$ and: $-8.7 \pm 21.8$, respectively) with significant RV dilatation $(\Delta \% \mathrm{RV}$ volume: $+10.6 \pm 9.9$ and $+15.3 \pm 12.8$, respectively). The RV basal segment made a major contribution to stroke volume during exercise, showing larger increases in size and strain as compared to the apex. Various patterns of RV adaptation to exercise, ranging from increases in both RV segmental strains and sizes to an insufficient increase in size and a decrease in strain, were identified; this individual variability was not correlated with prior training. Conclusions.-An acute $\mathrm{RV}$ impairment was demonstrated after a trail-running race, and was related to the amount of exercise. A high inter-individual variability was observed. Differences in RV adaptation patterns were independent of prior training, suggesting the influence was due to other individual factors.

\section{4:10-14:20}

Linear and Nonlinear Heart Rate Variability Following a 118-km Mountain Race

I. Martínez-Navarro, $\mathrm{PhD}^{1,3}$; O. Chiva-Bartoll, $\mathrm{PhD}^{2}$; E. Collado, $\mathrm{PhD}^{2}$; B. Hernando, $\mathrm{MSc}^{2}$; V. Porcar, $\mathrm{MD}^{2}$; N. Panizo, $\mathrm{PhD}^{2}$; A. Montoya-Vieco, $\mathrm{PhD}^{1,3}$; D. Sanmiguel, $\mathrm{MSc}^{3} ;$ C. Hernando, $\mathrm{PhD}^{2}$

${ }^{1}$ Universidad de Valencia, Departamento de Educación Física y Deportiva, Valencia, Spain, ${ }^{2}$ Universidad Jaume I, Castellon, Spain, ${ }^{3}$ Nisa Salud Deportiva, Hospital 9 de Octubre, Valencia, Spain
Objective.-This study examines changes in linear and nonlinear heart rate (HR) variability (HRV) following an ultraendurance mountain race. Moreover, as a secondary aim, possible relationships between finishing time and baseline HRV were also assessed. Methods.-Forty six athletes enrolled in the 2014 Penyagolosa Trails CSP115 race (118 km and a total positive elevation of $5439 \mathrm{~m}$ ) took part in the study. RR intervals were recorded the day before and immediately after the race using a Polar RS800 HR monitor and subsequently analyzed using HRV Analysis Software 2.0. HRV indices were compared before and after the race using Student's $t$-tests. Pearson correlation analyses were used to assess possible relationships between performance and prerace HRV data. Results.-Overall and vagally-mediated HRV (i.e. standard deviation of normal RR intervals, SDNN; root-mean-square difference of successive normal RR intervals, RMSSD) were significantly and largely decreased postrace $(3.70 \pm 0.45$ vs $3.10 \pm 0.56 \mathrm{~ms}, \mathrm{p}<.01$, ES $=-1.20 ; 3.63 \pm 0.56$ vs $2.77 \pm 0.69 \mathrm{~ms}$, p $<.01$, ES $=-1.39$ ). There was also a significant and large increase in HR fractal correlation properties (i.e., short-term scaling exponent, $\left.\alpha_{1}\right)(1.12 \pm$ 0.25 vs $1.43 \pm 0.24, \mathrm{p}<.01, \mathrm{ES}=1.32)$ jointly with a significant and large reduction in Sample Entropy (SampEn) $(1.71 \pm 0.27$ vs $1.48 \pm 0.28, p<$ $.01, \mathrm{ES}=-0.85)$. Besides, prerace SDNN and RMSSD were moderately and inversely correlated with finishing time $(r=-.46$ and $r=-.40 ; p<.05)$. Conclusions. - Our results showed that cardiac autonomic modulation became less complex and more predictable following the race (i.e., lower SampEn and higher $\alpha_{1}$ ). Concomitantly, postrace overall and vagally-mediated HRV diminishment coincided with previous studies involving mountain marathon races and ultraendurance events. Eventually, the relationship between prerace HRV and performance suggests that a better baseline profile of cardiac autonomic modulation contributes to a better performance.

Supported by NISA Hospitals group and "Cátedra Endavant Villarreal CF de l'Esport"

\section{4:20-14:30}

Monitoring Ultramarathon Runners Using Accelerometer Devices for Assessment of the Distribution of Physical Activity Intensity

Carlos Hernando, $\mathrm{PhD}^{1}$, Bárbara Hernando, $\mathrm{MSc}^{1}$, Ignacio MartinezNavarro, $\mathrm{PhD}^{2,3}$, Oscar Chiva, $\mathrm{PhD}^{2}$, Eladio Collado, $\mathrm{PhD}^{1}$, Nayara Panizo, $\mathrm{PhD}^{1}$, Vicente Porcar, $\mathrm{MD}^{1}$

${ }^{1}$ Jaume I University of Castellon, ${ }^{2}$ University of Valencia, ${ }^{3}$ Hospital NISA 9 de Octubre of Valencia

Objectives.- The purpose of this study was to use a triaxial accelerometer to monitor ultramarathon runners and to determine if runners who finished the race in the fastest times behaved differently in terms of effort distribu- 
tion compared to those finishers who achieved a slower time. Methods. Twenty seven finishers (23 males and 4 females), who participated in the 119-km Penyagolosa Trails CSP115 ultramarathon, wore a triaxial accelerometer from the night before to $168 \mathrm{~h}$ after the race. They were divided into two groups: faster runners $(\mathrm{FR} ; \mathrm{n}=13)$ and slower runners $(\mathrm{SR} ; \mathrm{n}=$ 14). Physical activity was analysed according to the four intensity categories previously validated. Results.-We did not observed differences in the overnight behaviour of FR compared with SR for all intensity categories $(p \geq .05)$. In the recovery week after the race, we only found differences in the first 24 hours for both sedentary and moderate intensity levels ( $p=.019$ and $\mathrm{p}=.048$; respectively). In the race segment, significant differences were observed between FR and SR for the four intensity categories: sedentary $(\mathrm{p}=.033)$, light $(\mathrm{p}=.003)$, moderate $\left(\mathrm{p}=4.20 \mathrm{E}^{-4}\right)$ and vigorous $(\mathrm{p}=1.72$ $\left.\mathrm{E}^{-4}\right)$. Conclusions. - The FR maintained high physical intensities along the race, running at vigorous intensity most of the race $(65.50 \pm 11.13 \%)$; while the SR showed a greater percentage of time racing at moderate intensity levels $(49.78 \pm 11.53 \%)$, with significant amount of time spent at light and sedentary levels. In summary, this study supports accelerometry as a useful technology for monitoring ultramarathon runners as well as for adjusting the effort distribution along the race in order to achieve performance goals.

Supported by the NISA Hospitals Group and the Villareal FC

\section{$14: 30-14: 40$}

Factors that Influence the Food Choices of Ultra-Endurance Athletes During Prolonged Training and Competition

Claire L Blennerhassett, $\mathrm{PgDip}^{1}$; Lars McNaugton, $\mathrm{PhD}^{2}$; S. Andy Sparks, $\mathrm{PhD}^{2}$

${ }^{1}$ Department of Health and Social Care, Edge Hill University, Ormskirk, UK, ${ }^{2}$ Department of Sport and Physical Activity, Edge Hill University, Ormskirk, UK

Objective.-Considerable energy deficits are commonly reported during ultra-endurance competition periods. This poses a potential risk to the performance and health of the athlete, especially for multi-day events. The aim of this study was to determine the factors that influence athlete's food choices in order to provide valuable insight into potential barriers to ingestion. Methods.-An online, validated food choices questionnaire (FCQ) was administered to recreational ultra-endurance athletes. The FCQ determined how important lifestyle and competition factors were on food choices during training and competition, using a 7-point Likert type scale $(1=$ extremely important and $7=$ extremely unimportant). Additionally, sources of nutrition information and advice were determined. Mann Whitney $\mathrm{U}$ tests were used to explore differences between runners and non-runners, high and low volume training, and gender. Results. - 102 ultra-endurance athletes completed the FCQ. This consisted of 69 runners, 33 non-runners (74 males and 28 females). No statistically significant differences were observed between groups in ratings of gut discomfort or time factors $(p>.09)$. The majority of athletes $(n=81)$ obtained nutrition information from several sources, favoring other athletes $(n=73)$ and magazines $(n=74)$ over nutritionists $(n=8)$. Factors most commonly rated as 'extremely important' to the athletes' choices were 'avoidance of gut discomfort' (62.4\%) and to 'provide energy' (52.5\%). Overall, the majority of factors that were rated as 'important' or 'extremely important' related to gut tolerability and logistical challenges associated with ultra-endurance competition, however $91.1 \%$ of athletes reported that it was also important that foods were nutritious. Despite considerable training loads ( $>10$ hours) for most of these athletes $(40 \%)$, 'time' and 'convenience' factors were more likely to be rated as unimportant. Conclusions.- Nutritionists need to actively engage with ultra-endurance athletes and support them to overcome the challenges of consuming sufficient nutritious foods and drinks to optimise energy availability, without causing discomfort.

\section{4:40-14:50}

\section{The Impact of Hydration Protocol "At Thirst" on Serum Sodium of Runners of the UTMB ${ }^{\circledR} 2015$}

Jean-Luc. Le Masle-Lastiolas, MD ${ }^{1}$; Patrick. Basset, $\mathrm{MD}^{2}$

${ }^{1}$ University of Nice, ${ }^{2}$ Ultra sports science

Objective.-Participation in ultra trail running is constantly increasing. Hyperhydratation is the main factor of exercise-associated hyponatremia (EAH) risk, which impact has reached $51 \%$ in certain ultratrails. Fortunately, most of these hyponatremics are asymptomatics, but a count of 14 deaths has already been directly related to the associated complications. Recently, the hydration strategy "drinking to thirst" has demonstrated its superiority in preventing EAH. However, these studies have been conducted mainly on desert races, with exceptional temperature and hygrometry conditions. The Natritrail study wanted to assess if this hydration protocol was strong and applicable to the UTMB $®$ race conditions. Methods.-The Natritrail study is a prospective, monocentric, single-blinded comparative trial of 196 runners divided into two groups, by their chosen hydration protocol "to thirst" or "another protocol." The main endpoint was the correlation between the change in serum sodium and the hydration protocol. Results.- The results of this study showed no superiority of the hydration protocol "at thirst" in serum sodium of runners. Runners from the "hydration to thirst" group tended to lose more weight than the others. No significant difference of characteristics, change in serum sodium, race results, or side effects were observed between the two groups. Conclusions.-EAH incidence was particularly low in this study, calculated at $1.5 \%$. This can be possibly explained by the exceptional temperatures of this edition, the altitude profile of the race and its impact on thirst but also by the many efforts put together to reduce the risks. However, the hydration to thirst, remains a good choice for EAH prevention. Promoting this approach is essential to ensure runner's health, as noted by the recommendations of the 2015 Carlsbad's consensus conference.

\section{4:50-15:00}

Does Vitamin D Status Influence the Exertional Stress Induced Cytokinaemia in Response to Ultra-Marathon Competition?

David Dixon, $\mathrm{MSc}^{1,2}$; Ricardo J.S. Costa, $\mathrm{PhD}^{3}$

${ }^{1}$ Coventry University, UK, ${ }^{2}$ University of East London, UK, ${ }^{3}$ Monash University, Australia

Objective.-Considering 56\% of the world's athletes are reported to have $\left(25(\mathrm{OH}) \mathrm{D}\right.$ status of less than $80 \mathrm{mmol} \cdot \mathrm{L}^{-1}$ and the potential role of $(25(\mathrm{OH})$ $\mathrm{D}$ in regulating pro- and anti-inflammatory cytokines, it is plausible that the $(25(\mathrm{OH}) \mathrm{D}$ status of ultra-endurance runners leading into competition may influence how cytokines respond to a situation of extreme exercise. The study aimed to determine whether vitamin $\mathrm{D}(25(\mathrm{OH}) \mathrm{D})$ status of ultra-endurance runners (UER, $n=12$ ) influences the pro- and anti-inflammatory cytokine profile in response to ultra-marathon competition. Methods.-Blood samples were taken before and immediately after the 24-hour ultramarathon (24h, 122$208 \mathrm{~km}$ ) and each stage of the $230 \mathrm{~km}$ multi-stage ultramarathon (MSUM). Samples were analysis for $25(\mathrm{OH}) \mathrm{D}$, C-reactive protein, IL-6, IL-1 $\beta$, TNF- $\alpha$, IFN- $\gamma$, IL-10, and IL-1ra concentrations. Results.-Suboptimal 25(OH)D levels were observed in $58 \%$ of UER $\left(62.8 \pm 5.6 \mathrm{nmol} \cdot \mathrm{L}^{-1}\right)$, while $42 \%$ of UER presented optimal 25(OH)D levels $\left(101.2 \pm 16.9 \mathrm{nmol} \cdot \mathrm{L}^{-1}\right)(\mathrm{p}=.001)$. The CON presented serum 25(OH)D concentration of $78.6 \pm 18.0 \mathrm{nmol} \cdot \mathrm{L}^{-1}$. A significantly greater pre to post competition increase in plasma CRP $(\mathrm{P}<$ 
$0.001)$, IL-6 $6(\mathrm{p}<.001)$ and IL-10 ( $=.003)$ concentrations was observed in the optimal and suboptimal $25(\mathrm{OH}) \mathrm{D}$ group compared with CON. No pre- to post-competition differences were seen between $25(\mathrm{OH}) \mathrm{D}$ groups and CON for IL- $1 \beta(\mathrm{p}=.091)$, TNF- $\alpha(\mathrm{p}=.058)$, and IFN- $\gamma(\mathrm{p}=.073)$ and there were no significant correlations observed between serum $25(\mathrm{OH}) \mathrm{D}$, $\mathrm{CRP}$ and cytokine responses. Conclusions. - The current study suggests that $25(\mathrm{OH}) \mathrm{D}$ status has little impact on cytokine responses to ultra-endurance running. It is likely that the sufficient levels of $25(\mathrm{OH}) \mathrm{D}$ within the athletes ( $>50 \mathrm{nmol} \cdot \mathrm{L}^{-1}$ ) were not low enough to promote cytokine perturbations and that other factors may play a more primary role in the perturbed cytokine profiles seen after extreme endurance exercise.

The study was funded by Coventry University Sport \& Exercise Science Applied Research Group and the Department of Health Professionals. Funded contributions were also provided by Coventry University as part of Dr. Ricardo Costa's Applied Research Fellowship and by University of East London as part of David Dixon's UEL Alumni 'Start up Research' grant.

\section{5:00-15:10}

Does Gender Influence Medical Requests on UTMB®? An Observational Study in 2015

E. Deman-Garin, Res. ${ }^{1}$; Sylvain Charreyre, MEng, Res. ${ }^{1}$; B. Basset ${ }^{2}$; P. Basset, $\mathrm{MD}^{3}$

${ }^{1}$ Emergency medicine department, CHU E. Herriot, Lyon, France, ${ }^{2}$ Dokever, CEO, Pierre-Bénite, France, ${ }^{3}$ Ultra Sports Science, President, PierreBénite, France

Objective.-Participants of the mythical foot raid Ultra Trail du Mont BlancUTMB ${ }^{\circledR}$ are exposed to severe weather conditions, important elevation gain $(>10,000 \mathrm{~m})$ and long running distance $(170 \mathrm{~km})$, in a remote environment. This study tends to identify whether gender varies the reason for medical requests. Methods.-Data were obtained from health records of the emergency stations (with doctors, nurses and/or first-aid) throughout the race in 2015. The primary diagnosis was analyzed: osteoarticular, cutaneous, digestive, discomfort/ exhaustion, neurological, temperature (hyper or hypo), respiratory, urinary, cardiological or ophthalmological related. Results.-Out of a total number of 2563 starters, 200 women (7.8\%) started the race. Global patient presentation rate (PPR) was $614 \%$ (1574 records), 147 regarding women and 1427 regarding men. PPR was respectively $735 \%$ and $604 \%$ o. Odds ratio (OR) (95\%) for men having medical requests was 0.72 [0.52-1.00]. The difference in PPR did not impact the race finishers percentage: $63.5 \%$ (1500) for men and 66\% (132) for women, OR 0.90 [0.66-1.21]. Osteoarticular issues represented for both groups nearly $40 \%$ of total complaints (M 39.9\%, W 38.8\%). Cutaneous problems were a little bit more frequent with men (M 33.3\%, W 28.5\%). Digestive issues represented the third reason for medical support in men $(9.8 \%)$ and the fourth in women $(8.8 \%)$, while discomfort/exhaustion represented the fourth in men (7.9\%) and third in women (15\%). Only discomfort/exhaustion was statically significant with a reduced probability with men. Conclusions.-Except for discomfort/exhaustion issues, which were less frequent with men, there was no differences in the 2 groups. These data are to be confirmed on other trails.

\section{$15: 10-15: 20$}

Trauma, Exposure Injuries and Illness During Two Ultramarathons in France: Observational Study

Sylvain Charreyre, MEng, Res. ${ }^{1}$; E. Deman-Garin, Res. ${ }^{1}$; B. Basset ${ }^{2}$; P. Basset, $\mathrm{MD}^{3}$

${ }^{1}$ Emergency medicine department, CHU E. Herriot, Lyon, France, ${ }^{2}$ Dokever, CEO, Pierre-Bénite, France, ${ }^{3}$ Ultra Sports Science, President, PierreBénite, France

Objective.-Participants of SaintéLyon (70 km) and UTMB® $(170 \mathrm{~km})$ ultramarathons are exposed to both difficult weather conditions and long running distances. The aim of the study was to identify the incidence of intrinsic pathologies observed related to the distance traveled. Methods.Data were obtained from health records of the emergency stations (with doctors, nurses and/or first-aid) throughout the races in 2015. The primary diagnosis was analyzed: osteoarticular, cutaneous, digestive, discomfort/ exhaustion, neurological, temperature (hyper or hypo), respiratory, urinary, cardiological or ophthalmological related. Results.-Out of a total number of 6020 runners of the SaintéLyon and 2563 of UTMB®, patient presentation rate was $35.7 \%$ (215) and 614\%o (1574), respectively. During UTMB®, osteoarticular (OA) issues (628 representing $40 \%$ of total complaints) were the primary reasons for medical request, followed by cutaneous (CU) problems $(524,33 \%)$. OA and CU complaints exploded after mid-race: 98 OA $(100 \mathrm{CU})$ requests cumulated at kilometer 80, $628(524 \mathrm{CU})$ at kilometer 170. Digestive issues represented the third reason for medical support (154, $10 \%$ ) but growth was linear throughout the race. For SaintéLyon, OA was also the major concern $(107,50 \%)$, followed by discomfort/exhaustion (57, $27 \%$ ) and digestive issues $(17,12 \%)$. Respiratory and cardiological issues were very rare at these ultra-trails. Conclusions.-These results will help

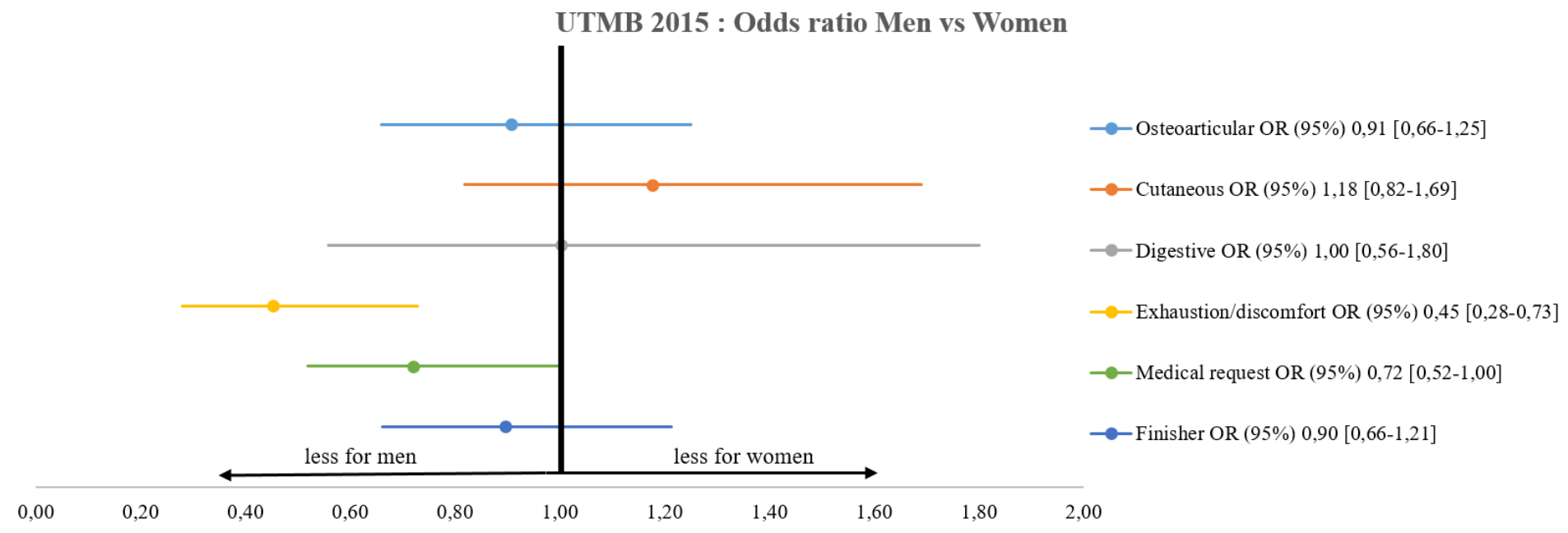

Figure 1 
medical teams staff the medical assistance teams according to the runners' needs in ultramarathons. It also will help event organizers to inform the runners what they need to be prepared for.

\section{$15: 20-15: 30$}

Resting Metabolic Rate, Somatotype and Body Composition of Trail Running Athletes

Samuel Amorim, MSc ${ }^{1,2}$; Rosa Costa, BSc${ }^{1}$; Ricardo Vardasca, $\mathrm{PhD}^{1,3}$; Marco Pontinha, MSc MD ${ }^{1,4}$

${ }^{1}$ Higiaclinic, ${ }^{2}$ Portuguese Cycling Federation, ${ }^{3}$ LABIOMEP, Faculty of Engineering, University of Porto, ${ }^{1}$ Hospital Senhora da Oliveira - Guimarães

Objective.-The purposes of this study were to analyze the resting metabolic rate (RMR), somatotype and body composition of trail runners and to explore if there are significant differences between runners from the two different types of races, trail and ultra-trail. This study also aimed to verify the applicability of RMR prediction equations in trail runners. Methods.-Thirty-two healthy Portuguese amateur male trail runners ( $36.2 \pm 7$ years old) were enrolled in this study. Anthropometric measurements were obtained by the same person, certified with level II course by the International Society for the Advancement of Kinanthropometry, and the RMR of each participant was obtained by indirect calorimetry. Results. - The RMR of trail runners $(2102.4 \pm 472.8$ kcal) was higher than ultra-trail runners $(1819.9 \pm 436.9 \mathrm{kcal})$, however it was not statistically significant. The RMR obtained by indirect calorimetry $(1987.6 \pm 472.8 \mathrm{kcal})$ was not statistical different from the RMR predicted by Cunningham equation $(1918.7 \pm 116.8 \mathrm{kcal})$. However, when RMR was predicted by Harris Benedict equation $(1666.7 \pm 124.2 \mathrm{kcal})$ the differences were statistically significant $(\mathrm{p}<.01)$. Mean somatotypes of 2.3-5.6-2.1 in trail runners (19 participants) and 2.4-5.3-2.1 in ultra-trail runners (13 participants) described both athletes as balanced mesomorphs. Greater values of body fat (\%) and sum of eight skinfolds were observed in trail runners compared with ultra-trail runners, without statistical significance. There were no statistical differences in other body composition results between trail and ultra-trail runners. Conclusions.-RMR is relevant to match the energy needs of daily training and competitive events of trail and ultra-trail runners. If possible, RMR should be obtained by indirect calorimetry. Otherwise, the RMR predicted by Cunningham equation can be acceptable.

\section{August 23, 2016}

\section{4:00-14:10}

Comparison of Two Intensities of Training on Body Composition and the Consumption of Oxygen in Ultrarunners

Guillermo Morea, MSc1 ; Gabriel Tarducci, PhD1; Ariel Bardach, $\mathrm{PhD}^{2}$

${ }^{1}$ University National of La Plata, IDHICS. Argentina, ${ }^{2}$ IECS, Buenos Aires, Argentina

Objective.-The intensity is an important component of exercise and its optimum magnitude can help to improve preparation for ultra-endurance sports performance. The objective of this study was to assess the effects of different intensities of exercise on body composition and the consumption of oxygen. Methods. - 20 healthy subjects were evaluated before and after a training program for 12 weeks in two groups; (a) moderate intensity and high volume training (MI), b) high-intensity and moderate volume of training (HI). The circumference of the waist, hip, body composition, anthropometric and maximum oxygen consumption were measured. Results.-Fat mass $(\mathrm{FM})$ decreased $2.8 \%$ in the MI group $(\mathrm{p}=.10)$. Muscle mass increased in both groups. Body weight did not differ significantly between groups.
The maximum oxygen consumption increased in the HI group $(\mathrm{p}<.05)$. MI modified somatotype by increasing the skeletal muscle component. Conclusions.-The MI training program produced further decline of the FM but not reaching statistical significance. The HI program improved significantly the consumption of oxygen. Combine training programs at different intensities could produce greater benefits in sports performance in the structural and functional profile of the ultrarunner.

\section{$14: 10-14: 20$}

Psycho-Physiological Responses to 21 Consecutive Half Marathons. A Case Study

Gianluca Rospo ${ }^{1}$; Francesco Sartor, $\mathrm{PhD}^{3}$; Matteo Bonato, $\mathrm{PhD}^{2}$; Antonio La Torre ${ }^{2}$

${ }^{1}$ School of Sport Science, Università degli studi di Milano, ${ }^{2}$ Department of Biomedical Sciences for Health, Università degli Studi di Milano, Milan Italy, ${ }^{3}$ Department of Personal Health, Philips Research, Eindhoven, The Netherlands

Objective.-To investigate the cardiac, autonomic, and psychological responses to 21 consecutive half-marathons in a middle-aged female nonprofessional athlete. Methods._A 42 years old woman (BMI: $19.8 \mathrm{Kg} / \mathrm{m}^{2}$; $\mathrm{VO}_{2 \max }: 45.9 \mathrm{ml} / \mathrm{kg} / \mathrm{min}$ ), performed 21 half-marathons in 21 consecutive days. Every morning, after awakening, heart rate variability (HRV) was recorded for $10 \mathrm{~min}$ in supine position. Inter-beat interval series spectral powers, indexes of vagal (HFnu; $0.15-0.40 \mathrm{~Hz}$ ), mixed para- and sympathetic tone (LFnu; 0.04-0.15 Hz) and sympatho-vagal balance (LF/HF ratio), were derived. A 32-items Profile of Mood States questionnaire, Total Quality Recovery (TQR) scale and Rating of Perceived Exertion (RPE) post exercise were collected daily. Results.-Running time throughout 21 consecutive events did not change significantly $(01: 54: 55 \pm 04: 55, \mathrm{p}=.44)$. HR rest $(\mathrm{r}=$ $-.7)$ and $\mathrm{LF} / \mathrm{HF}$ ratio $(\mathrm{r}=-.64)$ had a negative relation, and $\operatorname{LogRMSSD}(\mathrm{r}=$ $.65)$, pNN50 $(r=.56) \operatorname{LogHF} n u(r=.62)$ had a positive correlation with the number of consecutive running days, while it was biphasic for LogLFnu $(\mathrm{r}=$ .74). Energy index $(\mathrm{r}=-.87)$ and TQR scale $(\mathrm{r}=-.94)$ had a negative trend, while total mood $(\mathrm{r}=.8)$ and RPE post exercise $(\mathrm{r}=.62)$ resulted positive with the number of consecutive running days. Conclusions. - Twenty-one consecutive half-marathons seemed to have improved cardio-vagal control and sympatho-vagal balance, without a clear plateau suggesting a training effect. Psychological indexes seemed to reveal mental fatigue probably due to a lack of recovery days and training monotony. This case study suggested that monitoring HRV in parallel to psychological indexes could help to prevent a possible state of non-functional overreaching.

\section{$14: 20-14: 30$}

Pain Management, Cognitive Strength and Exercise Addiction in Ultramarathon Runners

Morteza Khodaee, MD, MPH ${ }^{1}$; Katherine Buck, PhD, LMFT ${ }^{2}$; Jack Spittler, MD, MS ${ }^{1}$; Heather Grothe, $\mathrm{MD}^{1}$; Anahita Saeidi, BS ${ }^{3}$; Alex Reed, PsyD, MPH ${ }^{1}$

${ }^{1}$ University of Colorado School of Medicine, Denver, Colorado, USA; ${ }^{2} J o h n$ Peter Smith Family Medicine Residency, Fort Worth, Texas, USA; ${ }^{3}$ Islamic Azad University, Karaj, Iran

Objective.-As the popularity of ultramarathons increases, there still exists a lack of understanding of the unique psychological characteristics of ultramarathon runners. The current study sought to investigate the specific emotional, behavioral, social, and cognitive factors involved in ultramarathoners. Methods. - The Bear Chase Trail Race is a single-day, 
multi-distance race consisting of a $10 \mathrm{~km}$, half-marathon, $50 \mathrm{~km}, 50$ mile, and $100 \mathrm{~km}$ run in Lakewood, Colorado at a base altitude of about $1680 \mathrm{~m}$. We obtained information via an online survey of runners of the $50 \mathrm{~km}, 50$ mile, and $100 \mathrm{~km}$ races in August of 2015. We administered the Exercise Addiction Inventory (EAI) and the PHQ-2 depression screening tool, and demographic information was collected. This information was analyzed and correlated with race finish times. Results.-Out of 200 runners who started the race, 98 (49\%) completed the survey. Over half of the runners were male $(60 \%)$ and average age was $39 \pm 10(21-77)$. Most respondents $(86 \%)$ held a bachelor's degree or higher and over half $(61 \%)$ reported having children. The majority of participants reported being married or in relationship with strong social support (with regard to ultramarathon running) from their current partner. A majority (88\%) reported they may experience pain, but they accept it as "they believe this is part of the race and this does not worry them." Over half of respondents (54\%) listed cognitive strength as the main factor that mentally aids them through a race, followed by quality of training. A large number of respondents (20\%) screened positive for exercise addiction (EAI $>24$ out of 30) and depression (PHQ-2 $\geq 2$ out of 6). Conclusions.-Our study population mirrors previously reported sample characteristics of ultramarathoners with regard to age, education level, and socioeconomic status. The relatively large number of positive screens on the EAI and PHQ-2 is alarming, even with good social support. Therefore, further investigation into exercise addiction and depression in the ultramarathon population is warranted.

\section{$14: 30-14: 40$}

Habits and Characteristics of the Ultrarunner in the Region of Northwest Argentina

Guillermo Morea, $\mathrm{MSc}^{1}$; Leonardo Isola ${ }^{2}$; Manuel Aybar, MSc ${ }^{3}$

${ }^{1}$ University National of La Plata, IDHICS, Argentina, ${ }^{2}$ Salteños Corren (trail magazine), Argentina, ${ }^{3}$ CONICET, Argentina

Objective.-Trail running is growing exponentially in Argentine and the world. Northwest Argentina is composed of the provinces of Salta, Jujuy, Tucumán, Catamarca, La Rioja and Santiago del Estero and presents a variety of biomes: high mountains, puna, valleys, gorges of the cordillera, saws sub-Andean and yungas to characterize landscapes often contrasting ranging from Andean cordilleras and pre mountain ranges bordering on $6000 \mathrm{~m}$. The objective of the study was to characterize the sports population and find associations with mountain ultramarathon performance. Methods.-We surveyed 90 trail runners from the northwestern region of the country to learn about training habits: volume of weekly kilometers, old sport, corridos ultramarathons, and ultramarathons finished. Results.-35\% participated in trail running races less than 2 years and $41 \%$ between 2 and 5 years. $41 \%$ had completed less than 5 ultra trails (UT), $18 \%$ ran successfully more than 10 UT and $29 \%$ prepared to compete in ultramarathons first. $59 \%$ of runners trained weekly between 30 and $59 \mathrm{~km}$ per week, while $35 \%$ trained between 60 and $90 \mathrm{~km}$ per week, and more than $90 \mathrm{~km}$ a week for $6 \%$ of the sample. There was a statistical association between those who trained more distance per week and who finished ultramarathons.

\section{4:40-14:50}

Acute Sleep Deprivation and Hallucinations in Ultra-Endurance Runners: A Descriptive Analysis During the Ultra-Trail du Mont-Blanc ${ }^{\circledR}$

Martin Dudoignon, MD student ${ }^{1}$; Renaud Jardri, MD, PhD/HDR ${ }^{2}$; Patrick Basset, $\mathrm{MD}^{3}$; Rémy Hurdiel, $\mathrm{PhD}^{4}$

${ }^{1}$ Henri Warembourg Medicine University, Lille 2 University, France, ${ }^{2}$ Univ Lille, CNRS UMR 9193, SCA-Lab \& CHU Lille, Psychiatry Dpt. (CURE platform), 59000 Lille, France, ${ }^{3}$ Dokever, Pierre-Bénite, France, ${ }^{4}$ Unité de Recherche Pluridisciplinaire Sport, Santé, Société - EA 7369, Université du Littoral Côte d'Opale, Dunkerque, France

Objective.-The objective was to determine the role of sleep deprivation in the occurrence of visual hallucinations $(\mathrm{VH})$ during a mountain ultramarathon (MUM). A secondary objective was to describe these psychotic symptoms. Methods.-Forty-eight non elite finishers of the UTMB ${ }^{\circledR} 2015$, all males, agreed to participate in the experiment. Immediately after they crossed the finish line, we administered a questionnaire related to (i) sleep strategy used during the race (time and duration of each sleep episode) and (ii) $\mathrm{VH}$ description. Items for $\mathrm{VH}$ description included frequency, duration, color, complexity, feeling of reality, control, and associated affective state. Results. $-56 \%$ (CI 95\% [42.2; 70.4]) of the participants experienced at least one VH episode. Two categories of sleep management were observed. Twenty-three finishers were "Sleepers" and 25 were "No Sleepers." Mean race durations were not different between the two groups (respectively $37.73 \pm 1.24 \mathrm{~h} v s 36.88 \pm 2.31 \mathrm{~h}$ ). "Sleepers" took $1.6 \pm 0.7$ naps of 10.4 $\pm 4.4 \mathrm{~min}$. Statistical analysis $\left(\chi^{2}\right)$ revealed an association between the "No sleepers" group and VH $(\mathrm{p}<.05) .84 \%$ of the "No sleepers" vs $26 \%$ of "Sleepers" experienced at least one $\mathrm{VH}\left(\chi^{2} ; \mathrm{p}=10^{-4}\right)$. No other association with hallucinatory experiences was found. However, 74\% (CI 95\% $[65.8 ; 82.2])$ of $\mathrm{VH}$ seem to be real and motionless black and white human beings. Conclusions.-Our study suggests a link between the severity of sleep deprivation and VH in MUM runners. Napping sleep strategy could be of interest to limit the onset of VH. Due to the small sample size of our study, we are planning a larger survey to explore the proneness to $\mathrm{VH}$ in sleep-deprived runners, identify safety/risk markers, and inform ultrarunners community about these at-risk phenomena.

\section{4:50-15:00}

No Evidence that Starting a 119-km Ultramarathon at Midnight Reduces Running Performance

Carlos Hernando, $\mathrm{PhD}^{1}$; Bárbara Hernando, $\mathrm{MSc}^{1}$; Ignacio MartinezNavarro, $\mathrm{PhD}^{2,3}$; Oscar Chiva, $\mathrm{PhD}^{2}$; Eladio Collado, $\mathrm{PhD}^{1}$; Nayara Panizo, $\mathrm{PhD}^{1}$; Vicente Porcar, $\mathrm{MD}^{1}$

${ }^{1}$ Jaume I University of Castellon, ${ }^{2}$ University of Valencia, ${ }^{3}$ Hospital NISA 9 de Octubre of Valencia

Objectives.-In order to avoid elevated temperatures, in the 2016 edition of the Penyagolosa Trails CSP115 race, runners started racing at $12 \mathrm{pm}$ rather than at 6 am like previous editions. The purpose of this study was to analyse the impact of starting the race at midnight on running performance, caused by a poor quality of rest before the ultramarathon. Methods.-Eight finishers (6 males and 2 females), who participated in both 2015 and 2016 editions of the 119-km Penyagolosa Trails CSP115 ultramarathon, wore a triaxial accelerometer from $9 \mathrm{~h}$ before the start time to the finish line. We compared the effort distribution of runners, in terms of physical activity intensity, between the accelerometer data collected in the 2015 and the 2016 race editions, according to the four intensity categories previously validated. Results. - During the pre-race time to rest, significant differences in the runners' behaviour were observed for sedentary $(64.88 \pm 11.14 \%$ in 2015 versus $41.48 \pm 16.33 \%$ in $2016, p=.005)$ and light $(24.00 \pm 8.25 \%$ in 2015 versus $43.13 \pm 10.87 \%$ in $2016, p=.001$ ) levels of physical activity. However, we did not observed differences in the effort distribution along the 119-km ultramarathon as well as in the racing intensity levels of each runner studied ( $\mathrm{p} \geq .05$ ). Additionally, running performances were similar in both race editions for all the runners (22:39 $\pm 2: 46$ hours in 2015 versus $21: 35 \pm 3: 32$ hours, $p=.52$ ). Conclusions. - We did not observe evidence of lower running performance as a consequence of starting a 119-km ultramarathon race at midnight, even though the quality of pre-race rest time 
was considerably poorer. This study suggests that running performance may possibly be more influenced by environmental factors, such as temperature or number of hours exposed to sunlight, than the quality of rest time the 9 hours prior to the ultramarathon race.

Supported by the NISA Hospitals Group and the Villareal FC

\section{5:00-15:10}

\section{Ultra-Endurance Sports Impact: Sportswomen Perceptions}

M. Delespierre, $\mathrm{MD}^{1}$; P. Basset, $\mathrm{MD}^{2}$; O. Dupuis, $\mathrm{PhD}^{3}$; JF. Luciani, $\mathrm{MD}^{4}$

${ }^{1}$ University of Lyon 1 Claude Bernard, France, ${ }^{2}$ Ultra Sports Science, President, Pierre-Bénite, France, ${ }^{3}$ Professor of university of Lyon sud, obstetrician gynecologist, France, ${ }^{4}$ Chief of sports medicine service, Edouard Herriot Hospital Lyon, France

Objective.-As soon as a physical practice is carried out intensively it may be harmful to physical and mental health. The complexity of the sports impact on a female athlete's body is even more important that it relates to intimacy: hormonal trouble, fertility, eating disorder. Our objective was to analyse the medical impacts of ultra endurance sport on sportswomen. Methods. - This was a qualitative study based on the grounded theory method, with semi-structured individual interviews oriented by a plan. The survey was conducted on sportswomen selected by theoretical sampling in the French area between June 2013 and August 2014. The interviews were transcribed, then vertically and transversely analysed, and then encoded. Results.-Fifteen interviews were conducted. Women recall both physical and mental pleasure when they talk about sports. Besides, they define themselves in opposition to male athletes by being about competitiveness, and rather about self-achievement. However, some negative aspects such as addiction or frustration due to injury are also reported. Moreover, hormonal disorders are unknown and trivialized by women. The practical aspect is taking over the health interests. We observed a mix between denial and misguiding about it. According to sportswomen, those disorders are mainly related to training hours, which make worrying about them irrelevant. The stress fracture associated with energy deficiency is unknown. Gear and training conditions are often wrongly incriminated. Conclusion.- The sportswomen evolve on a thin line between competitive behaviour towards optimization and pathological behaviour, harmful for the health. The main trouble source comes from a caloric deficiency. The doctor holds a central role in the support of those sportswomen. Indeed, they track down the disorders, and they guide them through a personalized and multidisciplinary caring process. A better prevention comes from adapted information about caloric deficiency disorders and risks related to unbalanced calories.

Supported by The Ultra Trail du Mont Blanc

\section{5:10-15:20}

\section{Is Response Inhibition Diminished Following a 118-km Mountain} Race?

I. Martínez-Navarro, $\mathrm{PhD}^{1,3}$; O. Chiva-Bartoll, $\mathrm{PhD}^{2}$; E. Collado, $\mathrm{PhD}^{2}$; B. Hernando, $\mathrm{MSc}^{2}$; V. Porcar, $\mathrm{MD}^{2} ;$ N. Panizo, $\mathrm{PhD}^{2}$; A. Montoya-Vieco, $\mathrm{PhD}^{1,3}$; D. Sanmiguel, $\mathrm{MSc}^{3}$; C. Hernando, $\mathrm{PhD}^{2}$

${ }^{1}$ Universidad de Valencia, Departamento de Educación Física y Deportiva, Valencia, Spain, ${ }^{2}$ Universidad Jaume I, Castellon, Spain, ${ }^{3}$ Nisa Salud Deportiva, Hospital 9 de Octubre, Valencia, Spain

Objective.-This study was aimed to assess whether response inhibition, a core component of cognitive executive function (EF), was diminished following an ultraendurance mountain race. Moreover, as a secondary aim, possible relationships between performance and response inhibition were also assessed. Methods.-Fifty recreational ultraendurance athletes participating in the 2015 Penyagolosa Trails CSP115 race $(118 \mathrm{~km}$ and a total positive elevation of $5439 \mathrm{~m}$ ) took part in the study. Response inhibition (i.e., the ability to suppress an over-learned response) was evaluated the day before the race and within $10 \mathrm{~min}$ after crossing the finishing line using the color-word interference task of the Stroop test (Golden version). Prerace and postrace scores were compared utilizing a Student's t-test. Pearson and partial correlation analyses were used to assess possible relationships between finishing time and Stroop performance (prerace and delta score, $\Delta$ ). Results. - Stroop performance did not change from prerace to postrace condition $(47.32 \pm 8.27$ vs. $46.29 \pm 7.52$ correct items; $p=.30)$. Correlation analyses showed that both prerace (using a partial correlation controlling for age differences) and $\Delta$ Stroop performance were unrelated to finishing time. Conclusions.-The absence of a significant decrease in EF after the race coincides with a former study carried out in a 100-h adventure race. However, other cognitive abilities such as psychomotor vigilance and choice reaction time have been shown to be altered following ultraendurance events. Therefore, it may be arguable that EF shows a greater resiliency than psychomotor vigilance and choice reaction time performance following an ultraendurance event. Further studies including a broader cognitive assessment are nevertheless required to verify this postulate.

Supported by NISA Hospitals group, "Cátedra Endavant Villarreal CF de l'Esport" and Sport HG 